\title{
Challenges of Patients with Neuromyelitis Optica Spectrum Disorder During COVID-19 Pandemic
}

\author{
(D) Serkan Ozakbas¹, (D) Cavid Baba², (D) Pelin Hancer2, (D) Ozge Sagici2, (D) Asiye Tuba Ozdogar2, (D) Zuhal Abasiyanik33, (D) Seda Dastan² \\ ${ }^{1}$ Dokuz Eylul University Faculty of Medicine, Department of Neurology, Izmir, Turkey \\ 2Dokuz Eylul University, Institute of Health Sciences, Department of Neurosciences, Izmir, Turkey \\ IIzmir Katip Celebi University Faculty of Health Sciences, Department of Physiotherapy and Rehabilitation, Izmir, Turkey
}

\section{Abstract}

Objective: At present, millions of people have been infected and hundreds of thousands are falling dead because of Coronavirus disease-2019. People with neuromyelitis optica spectrum disorder (NMOSD) are among patients who use immunosuppressive drugs.

Materials and Methods: Registered patients with NMOSD were contacted via phone during the three-months of pandemic.

Results: Information from 45 patients was gathered, and three (8.57\%) of them stopped their treatment due to fear for immunosuppression. All three were on rituximab treatment.

Conclusion: Infusion therapy was assumed to pose more fear in terms of immunosuppression on patients compared with noninfusion route of treatment.

Keywords: Multiple sclerosis, balance confidence, balance impairment, validity

\section{Introduction}

March 11, 2020 is a tragic date on which the Coronavirus disease-2019 (COVID-19) pandemic was recognized by the World Health Organization, stunning people globally (1). At present, millions of people have been infected, and hundreds of thousands are falling dead because of the virus (1). It is more stressful as it strikes the most vulnerable elders, people with chronic diseases, and those on immunosuppressants in addition to risk factors such as smoking and obesity (2-4). Demyelinating diseases get more attention for being chronic, with the usage of immunosuppressant treatments. Making definite conclusions is hard about drug usage and how doctors and people with risk factors should manage this issue due to a shortage of time because of the spread of disease (5). Diverse thoughts were presented regarding medicines used for treatment of multiple sclerosis (MS) and neuromyelitis optica spectrum disorders (NMOSD) as well as for the increased risk and protective effect during the pandemic $(5,6)$. All this information puts stress on patients diagnosed with NMOSD $(7,8)$. NMOSD is a condition that affects the elderly population, with more severe attacks and without an immunomodulator choice of treatment as in MS (9). In addition, patients face the risk of being infected during their visits to hospitals, which is an issue during attacks or routine visits (10). Considering all these factors against patients with NMOSD, which could pose difficulties to physicians actively involved in managing these patients (11), their condition was evaluated from their point of view; what are the patients' choices, how they managed the stress when the entire world swirled in the pandemic crisis, and how they deal with sedentary lifestyles during a lockdown.

\section{Materials and Methods}

This is a cross-sectional descriptive study of patients with NMOSD registered at a tertiary care center in Izmir, Turkey. Approval was obtained from The Ministry of Health of the Turkish Republic and the ethics committee of Dokuz Eylul University. The requirement for written consent was waived. A total of 71 patients with NMOSD registered at our clinic were included.

Address for Correspondence: Cavid Baba, Dokuz Eylul University, Institute of Health Sciences, Department of Neurosciences, Izmir, Turkey E-mail: cavidbaba@hotmail.com ORCID-ID: orcid.org/0000-0001-5455-7080

Received: 08.06.2021 Accepted: 02.08.2021

${ }^{\circ}$ Copyright 2021 by the Journal of Multiple Sclerosis Research published by Galenos Publishing House. 
Participants were interviewed by phone during work hours. Phone calls were made by the Multiple Sclerosis Study Group team of Dokuz Eylul University, including a physiotherapist, psychologist, nurse, and neurologist. The inclusion criterion includes their willingness to participate. The exclusion criterion was a severe cognitive decline, which will prevent verbal communication and comprehension.

Verbal consent was obtained from all participants before the interview. All participants were asked the same questions in the same order. From March 2020 to June 1, 2020, the Turkish Government applied lockdown on weekends, travel restrictions in some areas, temporary closure of most workplaces, recommendations to stay at home, social distancing, maskwearing, and other measures to decrease the risk of spreading COVID-19. This study aimed to evaluate patients in this timeframe. Out of 71 patients, a total of 45 were successfully reached out to. Of the 26 patients not included in this study, 5 did not want to participate and 21 were out of reach or did not answer the phone call; several attempts were made each time. Data regarding gender, age, disease duration, marital status, treatment, and information about the number of people they live together were collected. Next, questions related to COVID-19 pandemic were asked: their level of compliance with a stay at home recommendations; have they been tested positive for COVID-19; how the epidemic influenced their worries about NMOSD; the effect of lockdown in their routine controls, medical supply, and hospital visits for infusion treatment; were they able to practice their normal controls as scheduled; and what were their considerations about choices of treatment. All participants are registered in the Dokuz Eylul University database; thus, the latest expanded disability status scale (EDSS) for each patient were acquired from our registry. During the phone call, patients were asked about any changes in their condition related to NMOSD.

\section{Statistical Analyses}

Descriptive analyses were performed using the International Business Machines Statistical Package for the Social Sciences 26.0 software. Chi-square tests were used to compare categorical data. The significance level was set at $p<0.05$.

\section{Results}

Of the 71 registered patients with NMOSD, a total of 45 patients (37 female, 8 male) were successfully reached out to. The mean EDSS score of study participants was $2.31 \pm 2.32$ (range: 0-7.5) (Table 1).

Six patients had got tested for COVID-19, and none were positive. Four patients had stopped their treatment during the restriction period (Table 2 ).

Five patients (11.1\%) had decreased the time spent on physical exercises, six (13.3\%) spent more time on sportive activities compared with before the pandemic, and 10 (22.2\%) continued as usual. Before the lockdown, 10 patients did not use medication for NMOSD (patient's choice), 16 were on rituximab, 15 on azathioprine, and four on monthly methylprednisolone infusion. During the lockdown, three patients missed their routine control visits, two of them were on azathioprine, and one was receiving monthly methylprednisolone infusions (Table 3).

Out of 45 patients, only six (13.3\%) were afraid of contracting COVID-19 (Table 4).

Those on therapy were divided into two groups with infusion (rituximab and monthly methylprednisolone) and non-infusion therapies (azathioprine). Chi-square tests was performed to compare delayed therapy between patients receiving infusion versus noninfusion therapy, Fisher's Exact test $p=0.233$ (2-sided) (Table 5).

\begin{tabular}{|l|l|l|l|}
\hline \multicolumn{4}{|l|}{ Table 1. Patient characteristics } \\
\hline & N (45) & Mean & SD \\
\hline Sex & $\begin{array}{l}\text { F: } 37 \\
\text { M: } 8\end{array}$ & - & - \\
\hline Age & - & 43.82 & 12.42 \\
\hline EDSS & - & 2.31 & 2.32 \\
\hline Duration of disease & - & 7.87 & 8.04 \\
\hline
\end{tabular}

$\mathrm{N}$ : Number, SD: Standard deviation, EDSS: Expanded disability status scale

\begin{tabular}{|l|l|}
\hline \multicolumn{2}{|l|}{ Table 2. Delay in treatment } \\
\hline & N (\%) \\
\hline Issues with drug supply & $1(25)$ \\
\hline Fear of going out & $2(50)$ \\
\hline Pregnancy & $1(25)$ \\
\hline Total & $4(100)$ \\
\hline
\end{tabular}

Table 3. Delay in scheduled controls

\begin{tabular}{|l|l|}
\hline Fear of going out & N (\%) \\
\hline Canceled but had a phone-visit & $3(6.7)$ \\
\hline Travel restrictions & $7(15.6)$ \\
\hline No delay & $2(4.4)$ \\
\hline Total & $33(73.3)$ \\
\hline
\end{tabular}

Table 4. How pandemic influenced your considerations about NMOSD

\begin{tabular}{|l|l|}
\hline & N $(\%)$ \\
\hline More worried about contracting COVID-19 & $6(13.3)$ \\
\hline NMO could get worse & $15(33.4)$ \\
\hline No change/NMO is under control & $24(53.3)$ \\
\hline Total & $45(100)$ \\
\hline
\end{tabular}

COVID-19: Coronavirus disease-2019, NMO: Neuromyelitis optica, NMOSD: Neuromyelitis optica spectrum disorder 
Patients who were worried about contracting COVID-19 and those who were stressed about worsening the disease were categorized as a group with "worries," whereas others were grouped as "nonworried," and chi-square test was used to compare those who were on treatment versus those who were not. Continuity correction for chi-square came out as $p=0.100$, 2-sided (Table 6).

\begin{tabular}{|c|c|c|c|c|}
\hline Treatment optio & & & & \\
\hline \multirow{5}{*}{ Treatment delay } & \multicolumn{2}{|r|}{ Non-infusion } & Infusion & Total \\
\hline & \multirow[b]{2}{*}{ No delay } & Count 15 & 15 & 30 \\
\hline & & $\begin{array}{l}\text { Expected } \\
\text { count } 13.6\end{array}$ & 16.4 & 30.0 \\
\hline & \multirow[b]{2}{*}{ delay } & Count 0 & 3 & 3 \\
\hline & & $\begin{array}{l}\text { Expected } \\
\text { count } 1.4\end{array}$ & 1.6 & 3.0 \\
\hline \multirow{2}{*}{\multicolumn{2}{|c|}{ Total }} & Count 15 & 18 & 33 \\
\hline & & $\begin{array}{l}\text { Expected } \\
\text { count } 18.0\end{array}$ & 18.0 & 33.0 \\
\hline
\end{tabular}

\begin{tabular}{|c|c|c|c|c|}
\hline & & Disease ex & tation & \\
\hline \multirow{5}{*}{ Treatment } & & No worries & Worried & Total \\
\hline & \multirow{2}{*}{$\begin{array}{l}\text { No } \\
\text { treatment }\end{array}$} & Count 3 & 8 & 11 \\
\hline & & $\begin{array}{l}\text { Expected } \\
\text { count } 5.9\end{array}$ & 5.1 & 11.0 \\
\hline & \multirow[b]{2}{*}{ Treatment } & Count 21 & 13 & 34 \\
\hline & & $\begin{array}{l}\text { Expected } \\
\text { count } 18.1\end{array}$ & 15.9 & 34.0 \\
\hline \multirow{2}{*}{\multicolumn{2}{|c|}{ Total }} & Count 24 & 21 & 45 \\
\hline & & $\begin{array}{l}\text { Expected } \\
\text { count } 24.0\end{array}$ & 21.0 & 45.0 \\
\hline
\end{tabular}

\section{Discussion}

In this cross-sectional descriptive study, we tried to check if patients had stopped their treatment, how their drug supply was, how their scheduled hospital visits were managed, and how had the pandemic influenced their life regarding their disease. All registered patients diagnosed with NMOSD were contacted via phone. Out of the 71 registered patients, 45 were successfully reached out to. NMOSD has more severe attacks compared with MS, and incomplete recovery is another devastating feature that leads to accumulating disability with each exacerbation (12). Thus, preventive treatment is strongly recommended for all patients (13). Therefore, we expected that patients with NMOSD will be stricter with their treatment plan. Of those on treatment, only four (11.4\%) had stopped their medicine, of which three $(8.57 \%)$ were due to fear of immunosuppression. Comparing these results to patients with MS who stopped their treatment during the pandemic is necessary to see the actual influence of disease characteristics on adherence to therapy. During the three-month recommendation for not going out and weekend lockdown, visits were organized remotely via phone for patients who have scheduled controls on these days. A total of 12 participants had scheduled control during the lockdown. We managed to reach only seven of them. Two patients missed their control visit because of travel restrictions in their district, and the other three missed their scheduled control because of fear of going out. Unfortunately, they were not phone-visited, and two of them continued using azathioprine tablets. The other patient was on monthly methylprednisolone and missed it because of the fear of going out. Whether two patients on azathioprine have continued their treatment if they were on infusion therapy is unknown. Regardless, three patients demonstrated to be more worried about contracting COVID-19, and with a chance to stay out of contamination, they probably continued the treatment of NMOSD. This brings to light an issue of a safe environment for treatment and scheduled visits, and may include safe traveling to the hospital; it is an idea derived out of a small number of cases. Trials with more extensive sample data are warranted. Much speculation was observed about COVID-19 and immunosuppressant usage. Published literature mainly consists of cases showing various patient responses with use of immunosuppressant that contracted COVID-19 (5,6). This situation may agitate patients with NMOSD on treatment and force them to halt their ongoing treatment. The future regarding COVID-19 is unknown, but we know for sure that NMOSD would cause severe disability if left untreated. In addition, the biological activity of treatment choices available for NMOSD is delayed by 4-6 months after initiation (13). Making any conclusions about continuation of the treatment based on individual cases is unwise. Randomized clinical trials are necessary to validate these assumptions.

Among the therapy choices of our patients, 15 were on azathioprine, 16 on rituximab, 4 on methylprednisolone, and 10 without any maintenance treatment (patient's choice). All four patients who stopped their treatment were on rituximab treatment, and one stopped the treatment due to pregnancy. Patients could acknowledge infusion therapy as a more definitive treatment option that decreases immunity as opposed to tablets. The crosstabulation test showed no statistically significant result ( $p=0.233$ ) regarding the relationship between those who stopped their treatment and therapy options. This could be attributed to the small number of cases in the treatment delayed groups (3) and the total number of participants. The difference between those undergoing treatment and those who did not undergo treatment due to worries about COVID-19 and NMOSD were also evaluated, wherein any statistically significant result was not observed $(p=0.100)$, which could be attributed to the small number of participants. Limitations of 
our study include the small number of participants and the fact that $26(36.6 \%)$ patients were excluded. Thus, their influence on our results is unknown.

\section{Conclusion}

Results revealed that only those who were on infusion therapy stopped the treatment because they had no scheduled control during the lockdown and phone-visit could not be completed, which make us believe that people need more explanation from their doctors and be protected from the influence of social media or any information coming from sources other than their health institution. We consider this as our future reference in dealing with patients on infusion therapies, should any situation like lockdown or second wave of spread occur.

Acknowledgments: The authors acknowledge the Multiple Sclerosis Research Association for assistance during the recruitment of the study.

\section{Ethics}

Ethics Committee Approval: The research protocol was approved by Dokuz Eylul University Ethics Committee (date: 06.07.2020, code: 2020/17-26).

Informed Consent: Verbal informed consent was obtained prior to the interview.

\section{Authorship Contributions}

Concept: S.O., C.B., P.H., O.S., A.T.O., Z.A., S.D., Design: S.O., C.B., P.H., O.S., A.T.O., Z.A., S.D., Data Collection or Processing: S.O., C.B., P.H., O.S., A.T.O., Z.A., S.D., Analysis or Interpretation: S.O., C.B., P.H., O.S., A.T.O., Z.A., S.D., Literature Search: S.O., C.B., P.H., O.S., A.T.O., Z.A., S.D., Writing: S.O., C.B., P.H., O.S., A.T.O., Z.A., S.D.

Conflict of Interest: No conflict of interest was declared by the authors.

Financial Disclosure: The authors declared that this study received no financial support.

\section{References}

1. Coronavirus disease (COVID-19)weekly epidemiological update and weekly operational update. Available from: https://www.who.int/emergencies/ diseases/novel-coronavirus-2019/situation-reports/ (2020)
2. Wu C, Chen X, Cai Y, Xia J, Zhou X, Xu S, Huang H, Zhang L, Zhou X, Du C, Zhang Y, Song J, Wang S, Chao Y, Yang Z, Xu J, Zhou X, Chen D, Xiong W, Xu L, Zhou F, Jiang J, Bai C, Zheng J, Song Y. Risk Factors Associated With Acute Respiratory Distress Syndrome and Death in Patients With Coronavirus Disease 2019 Pneumonia in Wuhan, China. JAMA Intern Med 2020;180:934943.

3. Ruan Q, Yang K, Wang W, Jiang L, Song J. Clinical predictors of mortality due to COVID-19 based on an analysis of data of 150 patients from Wuhan, China Intensive Care Med. 2020 doi: 10.1007/s00134-020-05991-x.

4. Chen G, Wu D, Guo W, Cao Y, Huang D, Wang H, Wang T, Zhang X, Chen H, Yu H, Zhang X, Zhang M, Wu S, Song J, Chen T, Han M, Li S, Luo X, Zhao J, Ning Q. Clinical and immunological features of severe and moderate coronavirus disease 2019. J Clin Invest 2020;130:2620-2629.

5. Giovannoni G, Hawkes C, Lechner-Scott J, Levy M, Waubant E, Gold J. The COVID-19 pandemic and the use of MS disease-modifying therapies. Mult Scler Relat Disord 2020;39:102073. doi: 10.1016/j.msard.2020.102073.

6. Montero-Escribano P, Matías-Guiu J, Gómez-Iglesias P, Porta-Etessam J, Pytel V, Matias-Guiu JA. Anti-CD20 and COVID-19 in multiple sclerosis and related disorders: A case series of 60 patients from Madrid, Spain. Mult Scler Relat Disord 2020;42:102185. https://doi.org/10.1016/j.msard.2020.102185.

7. Barzegar M, Badihian S, Mirmosayyeb O, Ashtari F, Jamadi M, Emami S, Jahani L, Safavi A, Shaygannejad V. Comparative study of quality of life, anxiety, depression, and fatigue among patients with neuromyelitis optica spectrum disorder and multiple sclerosis: The first report from Iran. Mult Scler Relat Disord 2018;22:161-165.

8. Shi Z, Chen H, Lian Z, Liu J, Feng H, Zhou H. Factors that impact healthrelated quality of life in neuromyelitis optica spectrum disorder: anxiety, disability, fatigue and depression. J Neuroimmunol 2016;293:54-58.

9. Palace J, Lin DY, Zeng D. Outcome prediction models in AQP4-IGG positive neuromyelitis optica spectrum disorders. Brain 2019;142:1310-1323.

10. Wang D, Hu B, Hu C, Zhu F, Liu X, Zhang J, Wang B, Xiang H, Cheng Z, Xiong Y, Zhao Y, Li Y, Wang X, Peng Z. Clinical characteristics of 138 hospitalized patients with 2019 novel coronavirus-Infected pneumonia in Wuhan, China. JAMA 2020;323:1061-1069. Erratum in: JAMA 2021;325:1113.

11. Salama S, Giovannoni G, Hawkes CH, Lechner-Scott J, Waubant E, Levy M. Changes in patient and physician attitudes resulting from COVID-19 in neuromyelitis optica spectrum disorder and multiple sclerosis. Mult Scler Relat Disord 2020;42:102259. doi:10.1016/j.msard.2020.102259.

12. Wingerchuk DM, Hogancamp WF, O'Brien PC, Weinshenker BG. The clinical course of neuromyelitis optica (Devic's syndrome). Neurology 1999;53:11071114.

13. Weinshenker BG, Wingerchuk DM. Neuromyelitis spectrum disorders. Mayo Clin Proc 2017;92:663-679. 